Supplementary Information

\title{
Optoelectronics and Nanophotonics of Vapor-Liquid-Solid Grown GaSe van der Waals Nanoribbons
}

\author{
Peter Sutter, ${ }^{1}$ Jacob S. French, ${ }^{1}$ Larousse Khosravi Khorashad, ${ }^{1}$ Christos Argyropoulos, ${ }^{1}$ \\ and Eli Sutter ${ }^{2}$ \\ ${ }^{1}$ Department of Electrical \& Computer Engineering, University of Nebraska-Lincoln, \\ Lincoln, Nebraska 68588, United States \\ ${ }^{2}$ Department of Mechanical \& Materials Engineering, University of Nebraska-Lincoln, \\ Lincoln, Nebraska 68588, United States
}

\section{Materials and Methods}

Synthesis of GaSe nanoribbons: GaSe nanoribbons were synthesized by vapor-liquidsolid growth involving vapor transport from stoichiometric GaSe powder (99.999\%, ALB Materials) in a quartz tube reactor with two independently controlled temperature zones. $\mathrm{Si}$ and $\mathrm{SiO}_{2} / \mathrm{Si}$ wafers covered by thin $(2-4 \mathrm{~nm}$ ) Au or Ag films (dewetted at the growth temperature) were used as substrates. Zone 1 of the reactor containing a quartz boat with GaSe powder $(50 \mathrm{mg})$ was heated to $850^{\circ} \mathrm{C}$, while the sample held in Zone 2 was heated to $500-600^{\circ} \mathrm{C}$. The reactor was pumped to $10^{-3}$ Torr and $\operatorname{Ar}(99.9999 \%$, Matheson) carrier gas flow was maintained at $60 \mathrm{sccm}$. The typical growth duration was 10 minutes, after which the reactor was cooled naturally.

Optical microscopy, confocal Raman and PL spectroscopy, atomic force microscopy: Optical imaging, micro-Raman and micro-PL spectroscopy were performed on a Horiba XPlora Plus microscope using 50× and $100 \times$ objectives. Large-area optical images were acquired with a $50 \times$ objective, by software controlled scanning of the sample and automated stitching of individual images across overall fields of view up to $2 \mathrm{~mm}$. For larg-scale optical microscopy as well as atomic force microscopy (AFM) imaging, GaSe nanoribbons were dry-transferred from forests on the growth substrates onto flat $\mathrm{SiN}_{\mathrm{x}} / \mathrm{Si}$ chips (Norcada). Raman and PL spectra were obtained with laser excitation at $532 \mathrm{~nm}$ and typical power of $0.168 \mu \mathrm{W}$, using gratings with 1800 and 600 lines per mm, respectively. PL maps were acquired by scanning the sample with typical pixel integration time of $50 \mathrm{~ms}$. AFM was performed in tapping mode on a Veeco Multimode AFM with Nanoscope V controller, and using Si cantilevers with $300 \mathrm{kHz}$ resonance frequency (Budget Sensors Tap300Al).

Electron microscopy and STEM cathodoluminescence (CL): Structure and morphology of the nanoribbons were analyzed by (scanning) transmission electron microscopy ((S)TEM) following dry-transfer from the growth substrate to lacey carbon TEM grids. TEM imaging, diffraction, and STEM-CL were performed in an FEI Talos F200X microscope at $200 \mathrm{keV}$ electron energy. STEM-CL was performed using a Gatan Vulcan 
holder at room temperature, with incident electron beam currents of $\sim 400 \mathrm{pA}$. In panchromatic CL maps (512×512 pixels, $2 \mathrm{~ms}$ per pixel), broadband (400-1000 nm) light was detected by a Peltier-cooled photomultiplier detector. CL spectra were obtained using a $300 \mathrm{~mm}$ Czerny-Turner spectrometer and a cooled back-illuminated Si CCD detector. Hyperspectral linescans were acquired by positioning the focused electron beam in equal steps along the indicated paths and acquiring full CL spectra at each beam position (Integration time $10 \mathrm{~s}$ per spectrum).

Numerical simulations: Numerical calculations were performed for one-dimensional GaSe nanoribbons whose geometry in the simulations was chosen to match the nanoribbon sizes and shapes observed experimentally. Field profiles inside and field decay lengths outside GaSe nanoribbon waveguides were numerically calculated via fullfield 3D and 2D simulations in the frequency domain (Comsol Multiphysics), using the published bulk permittivity of $\mathrm{GaSe}^{1-3}$ and with simulated ribbon dimensions matching those in the experiments. The results of 3D simulations are shown in Figure 5 (main paper) and Figure S14. The results of 2D simulations are depicted in Figure S16 and Figure S19. The entire geometry of the nanoribbon was surrounded by vacuum/air as the ambient medium in both 3D and 2D simulations. To reduce the computational time, we used a 2D model with transverse electric (TE) polarized port excitation to compute the propagating mode in the GaSe waveguide, with results shown in Figure S16. The 2D simulated propagating mode has been compared with $3 \mathrm{D}$ simulations and the results were found to be similar. Scattering boundary conditions were used in all simulations to absorb the total power outflow and prevent backscattering from the boundaries of the simulation domain. To compute the induced electric fields in GaSe waveguides due to the electron beam passage, 3D simulations were performed where the beam was simulated by an aloof line current (positioned in vacuum/air, $10 \mathrm{~nm}$ away from the nanoribbon). ${ }^{4}$ The relevant results of these calculations are shown in Figure 5 (main paper) and Figure S14. These 3D simulation calculations were carried out with and without the GaSe waveguide using the same mesh. Finally, 2D simulations were used to compute the evanescent decay lengths by placing a TE polarized port adjacent to one end of the waveguide and calculating the field in the cladding vacuum/air for different energies as a function of distance from the waveguide. ${ }^{4}$ These 2D simulation results are shown in Figure S19 (e) and found to be in good agreement with the corresponding experimental results. 


\section{Supplementary Figures}
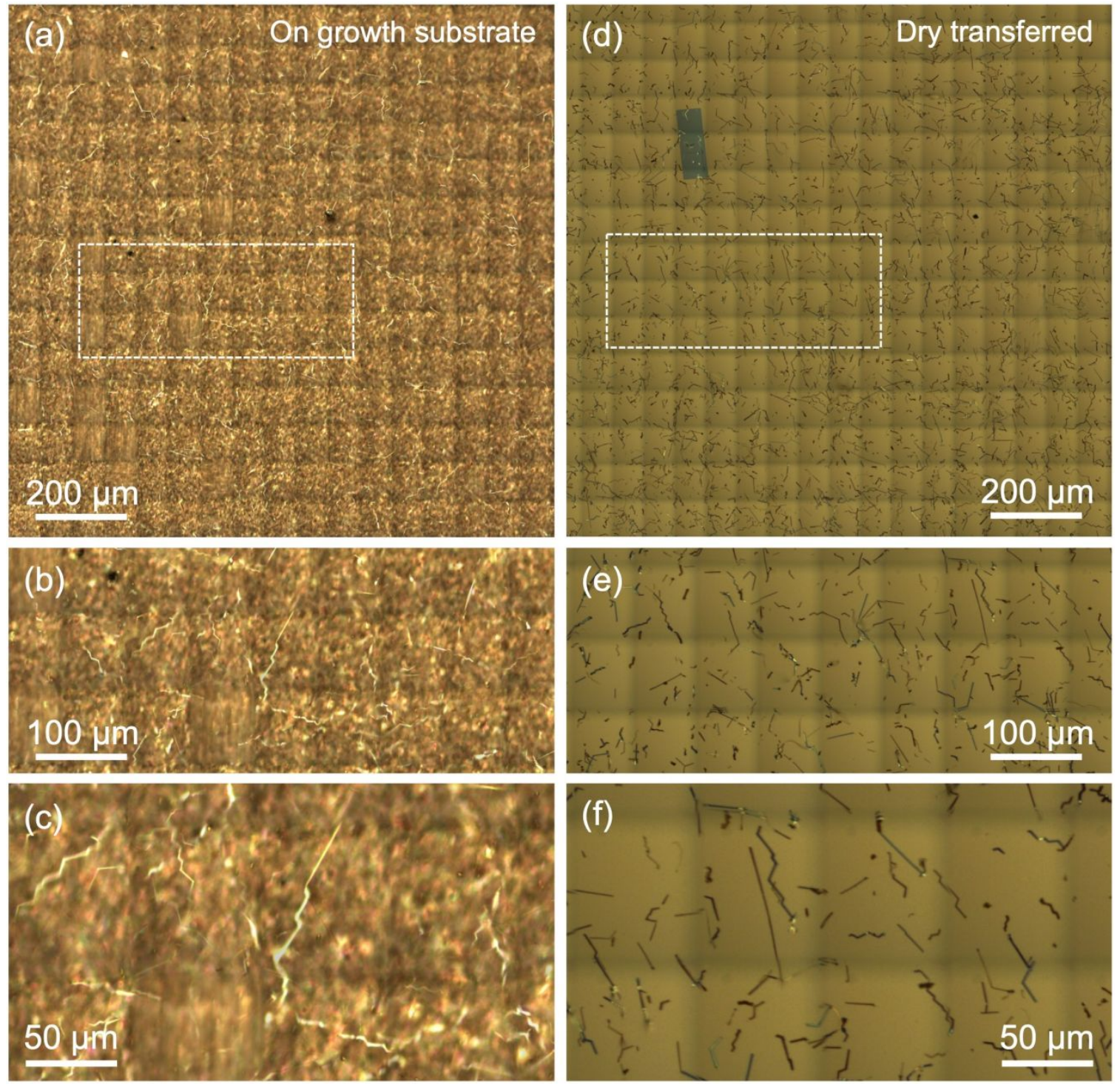

Figure S1. Morphology of forests of GaSe nanoribbons on the growth substrate (left) and after dry transfer to $\mathrm{SiN}_{\mathrm{x}}$ support. Growth conditions: $500^{\circ} \mathrm{C}$ over Au VLS catalyst. (a) - (c) Optical microscopy images of the GaSe nanoribbons on the Au/Si growth substrate. All areas are covered with vertical ribbons that lie mostly outside the focal plane of the microscopy objective, while some ribbons grown parallel to the substrate surface are in focus over long distances. (d) (f) Optical microscopy images of the GaSe nanoribbons from the same sample, following dry transfer onto a $\mathrm{SiN}_{\mathrm{x}} / \mathrm{Si}$ chip (Norcada Inc.). Note the high density of nanoribbons, consistent with the high density observed on the native growth substrate. 


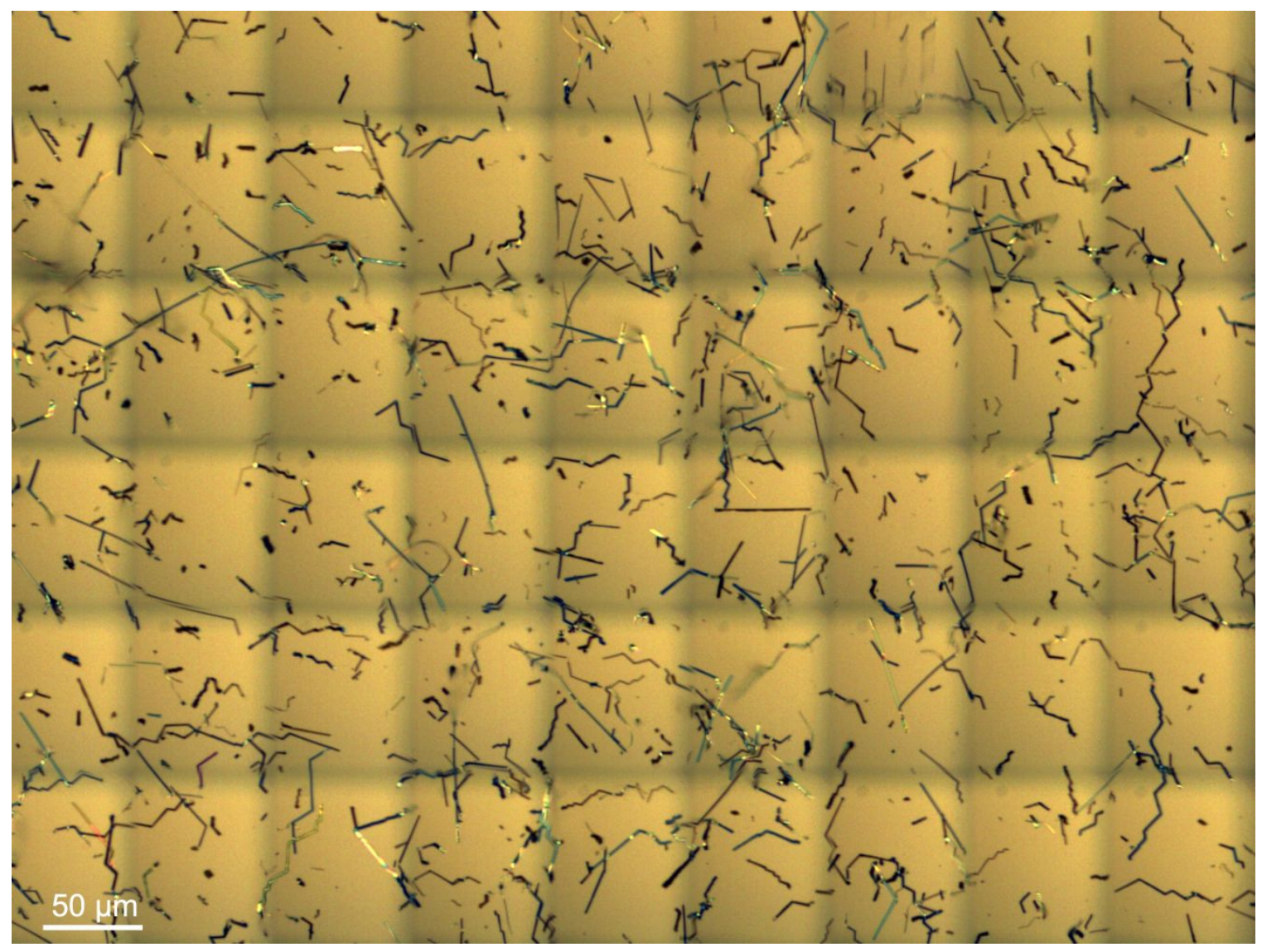

Figure S2. GaSe nanoribbons after dry transfer to $\mathrm{SiN}_{\mathrm{x}}$ support. Growth conditions: $500^{\circ} \mathrm{C}$ over Au VLS catalyst. Optical microscopy image of the GaSe nanoribbons following dry transfer onto a $\mathrm{SiN}_{\mathrm{x}} / \mathrm{Si}$ chip (Norcada Inc.). Two populations of nanoribbons exist, one of which shows multiple kinks between straight segments with typical length of a few $\mu \mathrm{m}$ to several tens of $\mu \mathrm{m}$, whereas the other reaches lengths of over $100 \mu \mathrm{m}$ without any direction changes. 

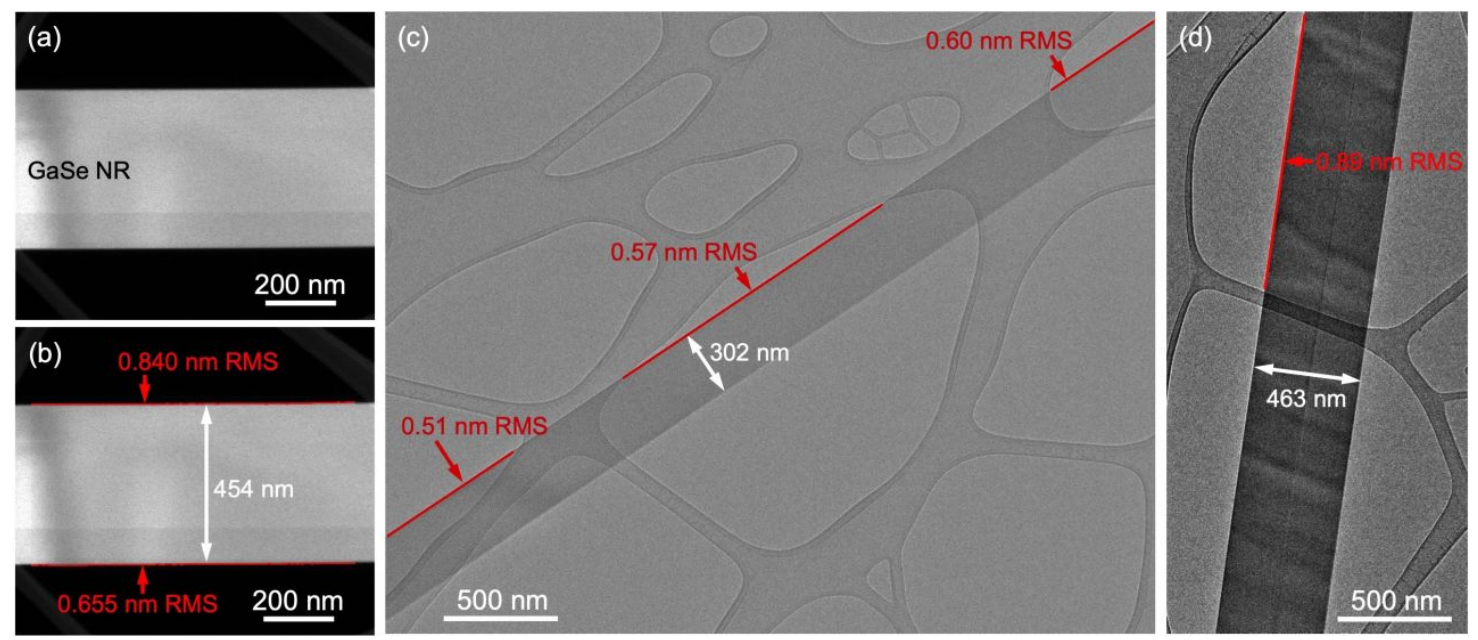

Figure S3. Edge morphology of low-temperature $\left(500^{\circ} \mathrm{C}\right)$ vapor-liquid-solid (VLS) synthesized GaSe nanoribbons using Au catalyst. (a) STEM image of a section of a GaSe nanoribbon of $454 \mathrm{~nm}$ width, grown at $500^{\circ} \mathrm{C}$ substrate temperature with Au catalyst. (b) Analysis of the root-mean-square (RMS) roughness of the nanoribbon edges, using ImageJ and the "AnalyzeStripes" macro (version 2.6, see https://imagej.net/Analyze_Stripes). (c) TEM image of a GaSe nanoribbon grown at $500^{\circ} \mathrm{C}$ substrate temperature with Au catalyst, again with RMS edge roughness values given for $\mu \mathrm{m}$ long sections. (d) TEM image of another GaSe ribbon grown under the same conditions with edge roughness analysis. All RMS edge roughness values in (b)(d) were obtained on raw (S)TEM images without any filtering, smoothing, etc.
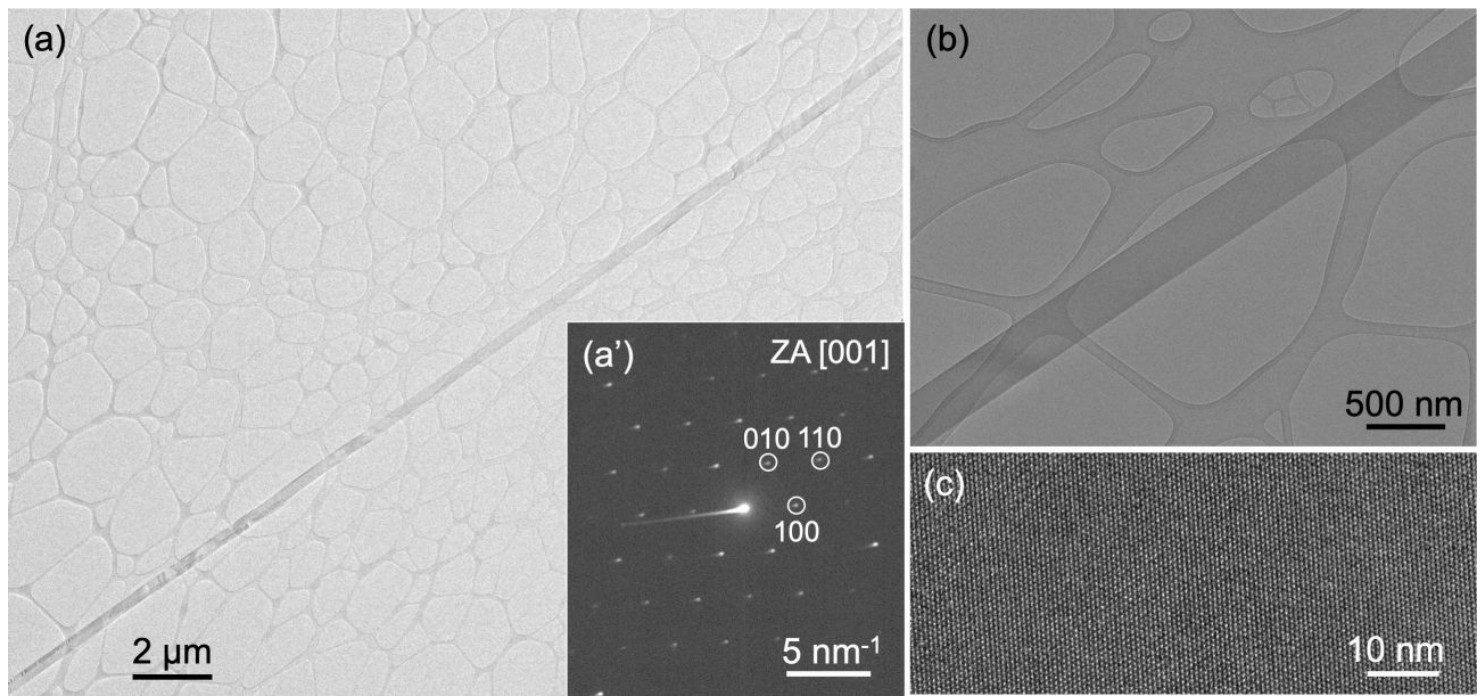

Figure S4. TEM of a straight GaSe nanoribbon grown by VLS over Au catalyst at $500^{\circ} \mathrm{C}$. (a) Overview TEM image of a long, straight section of the nanoribbon. (a') Electron diffraction pattern indexed to GaSe along [001] zone axis (ZA). Note the orientation of the ribbon axis along the [110] axis in reciprocal space, which for the hexagonal GaSe structure corresponds to a armchair ribbon edge termination. (b) Higher magnification view, showing the uniform ribbon width and smooth edges. (c) High-resolution TEM image of the basal-plane oriented GaSe nanoribbon. 


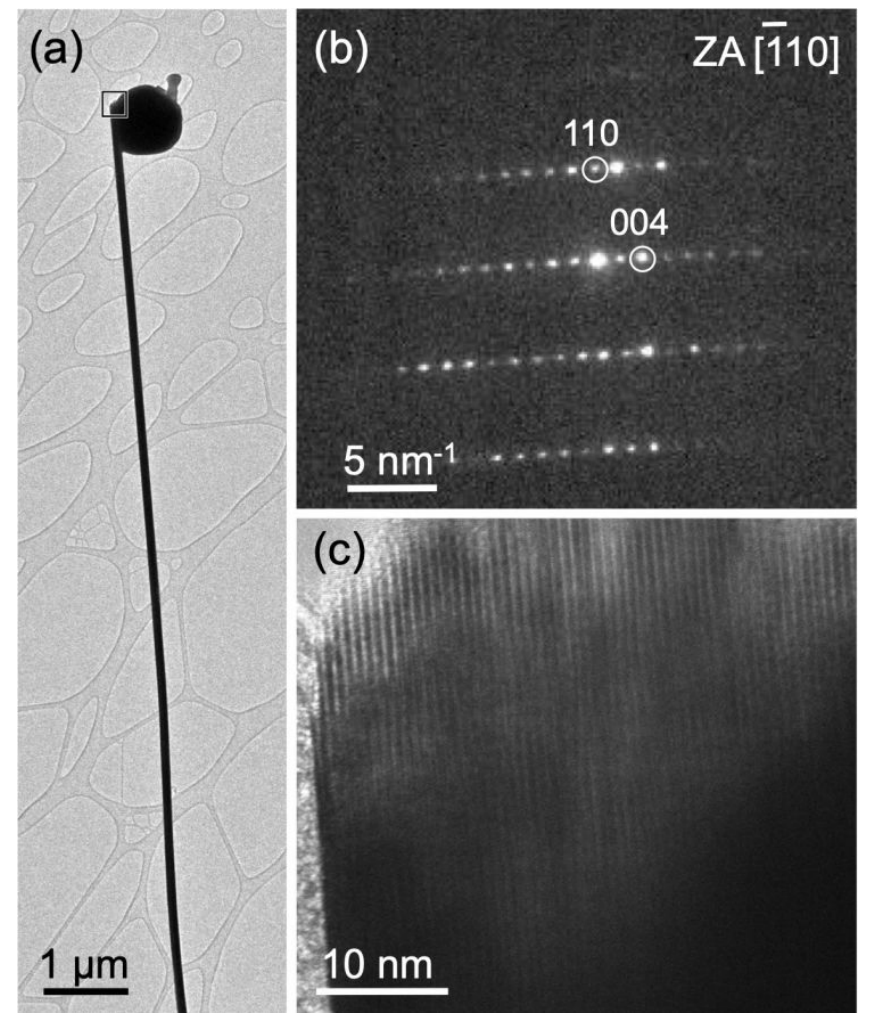

Figure S5. TEM of a standing GaSe nanoribbon supported by its VLS catalyst. (a) TEM image of the vertically standing nanoribbon, carrying a large VLS catalyst particle at its tip. Growth temperature: $500^{\circ} \mathrm{C}$; Au VLS catalyst; Si substrate. (b) Selected-area electron diffraction pattern (zone axis (ZA) [110]). Closely spaced reciprocal space reflections indicate the layering direction of the GaSe van der Waals crystal. (c) High-resolution TEM image obtained at the interface between the GaSe nanoribbon and its VLS catalyst.
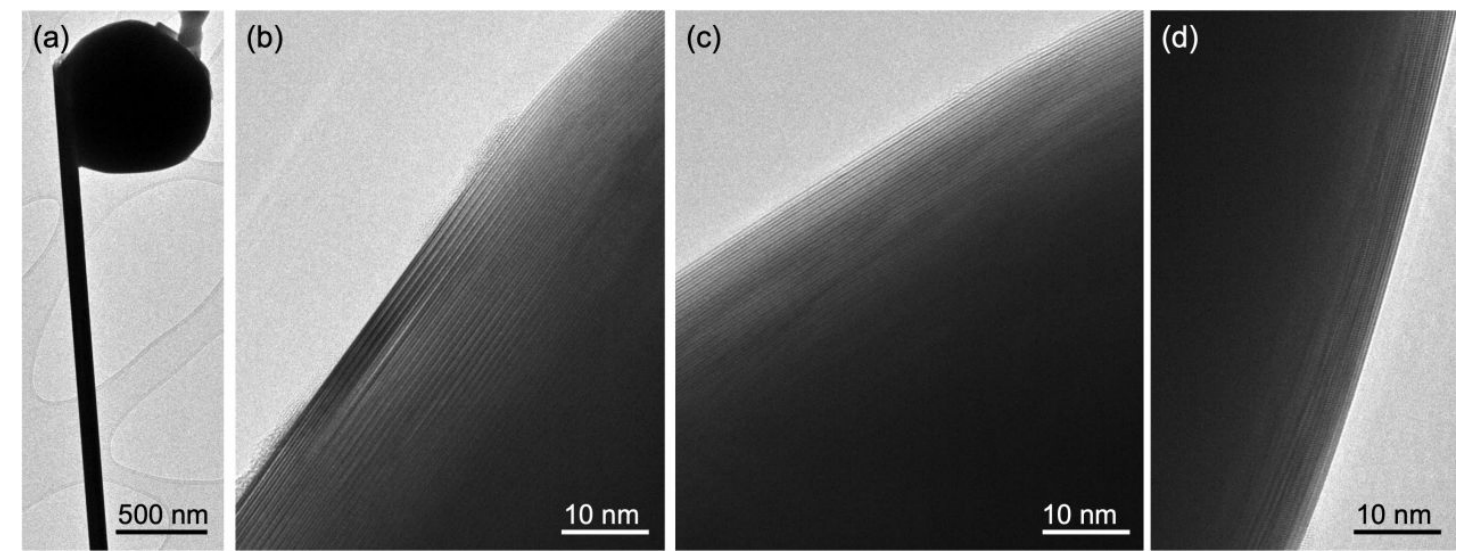

Figure S6. TEM of the crystallized VLS catalyst of a standing GaSe nanoribbon. (a) TEM image of the vertically standing nanoribbon near its tip, which carries a large VLS catalyst particle. Growth temperature: $500^{\circ} \mathrm{C}$; Au VLS catalyst; Si substrate. (b)-(d) High-resolution TEM images of different near-surface regions of the VLS catalyst, showing its encapsulation in a layered GaSe shell. 
(a)
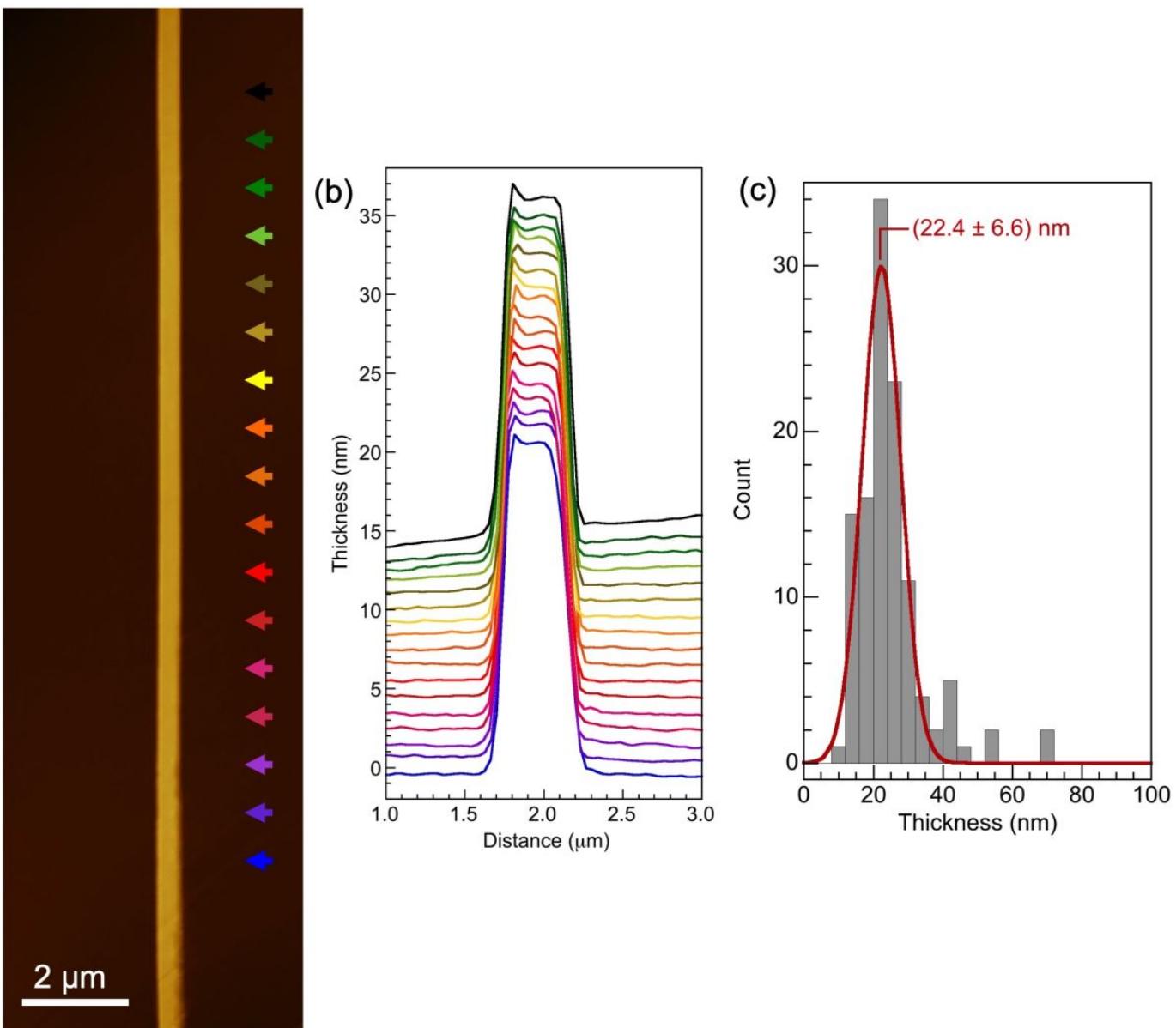

Figure S7. AFM characterization of GaSe nanoribbons, grown at $500^{\circ} \mathrm{C}$ over $\mathrm{Au}$ VLS catalyst and dry-transferred onto a $\mathrm{SiN}_{\mathrm{x}} / \mathrm{Si}$ chip (see Figs. S1-S2). (a) AFM image of a straight nanoribbon. (e) Series of thickness profiles obtained at regular intervals along the ribbon (colored arrows in (a)). (f) Histogram of the thickness distribution of 115 nanoribbons (grown at $500^{\circ} \mathrm{C}$ over Au VLS catalyst, see Figures S1-S2) imaged by AFM. Mean thickness under these conditions: $22.4 \mathrm{~nm}$ ( 28 GaSe layers). No nanoribbons with thickness exceeding $100 \mathrm{~nm}$ were found on this sample. 

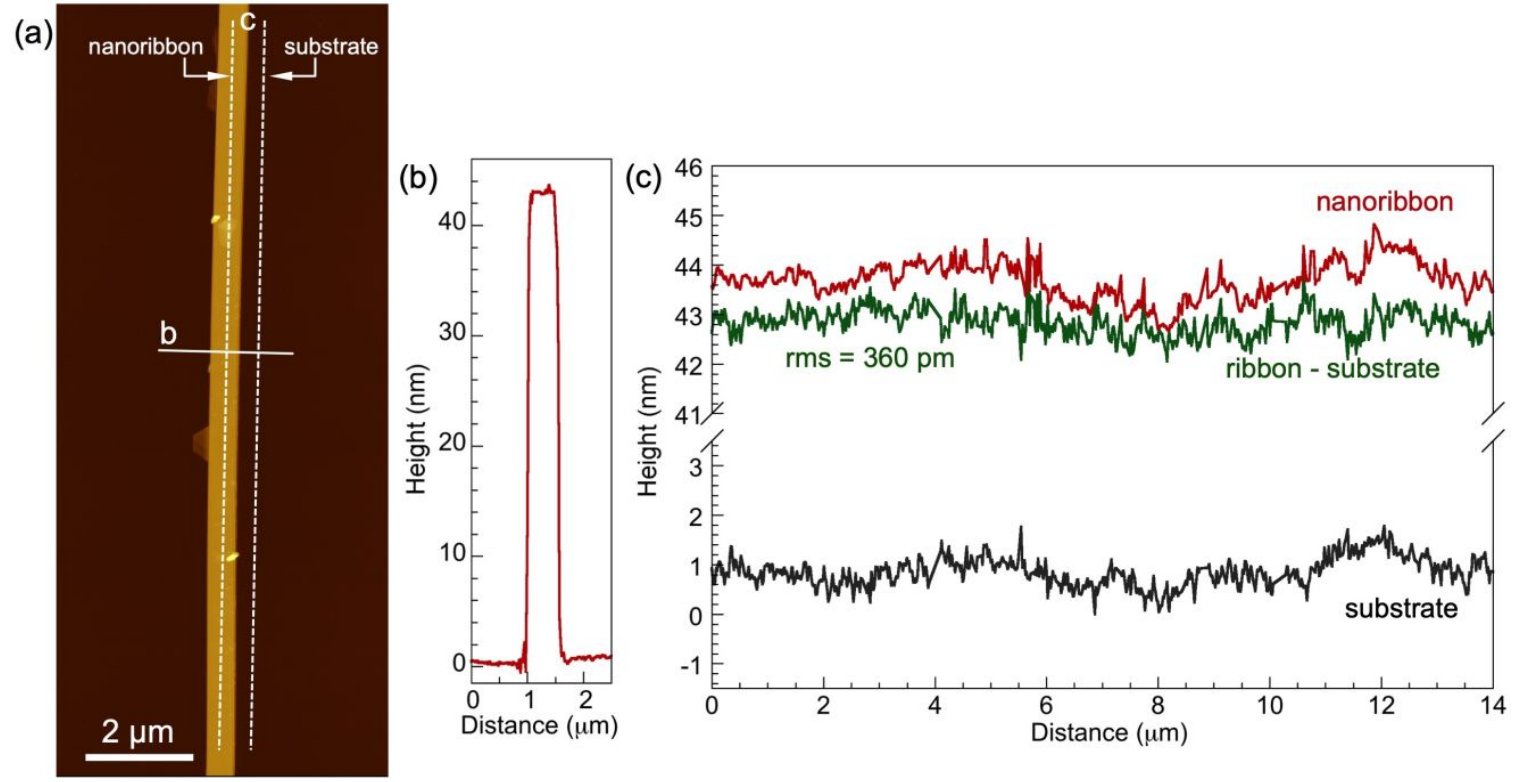

Figure S8. AFM measurement of surface roughness/thickness variations of GaSe nanoribbons grown at $500^{\circ} \mathrm{C}$ over Au VLS catalyst and dry-transferred onto a $\mathrm{SiN}_{\mathrm{x}} / \mathrm{Si}$ chip (see Figs. S1-S2). (a) AFM image of a straight GaSe nanoribbon with $\sim 44 \mathrm{~nm}$ thickness. Line profiles were obtained across the ribbon (b), and along the nanoribbon as well as on the substrate (c). (b) Transverse height profile along the solid line in (a). (c) Longitudinal line profiles on the nanoribbon (red) and on the adjacent $\mathrm{SiN}_{\mathrm{x}} / \mathrm{Si}$ substrate (black). The nanoribbon clearly follows long-range height variations of the underlying substrate. Short-range peaks and valleys correspond to z-noise of our AFM scanner. The green trace is the height profile of the nanoribbon from which the profile of the substrate has been subtracted. Here, analysis shows root-meansquare (RMS) height variations of $\sim 0.36 \mathrm{~nm}$, which is less than $50 \%$ of the thickness of a single GaSe monolayer (Se-Ga-Ga-Se, $\sim 0.8 \mathrm{~nm}$ ). Hence, the GaSe nanoribbons are atomically smooth with uniform thickness (i.e., no monolayer thickness variations; 1 Se-Ga-Ga-Se layer $\sim 0.8 \mathrm{~nm}$ ) over lengths of at least $15 \mu \mathrm{m}$. 


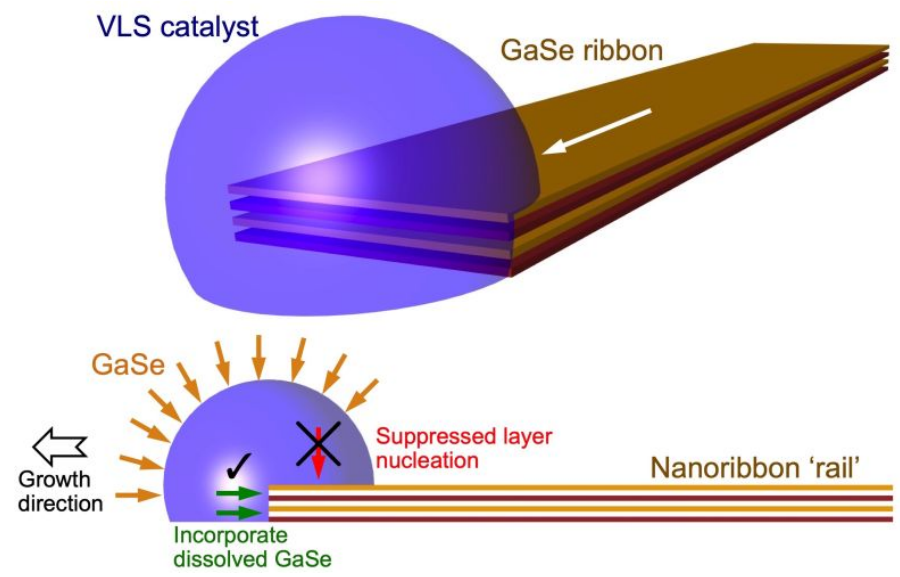

Figure S9. Schematic of the inferred VLS GaSe nanoribbon growth mode. The VLS catalyst is supported by one of the (001) basal planes (e.g., at the top of the ribbon) and overlaps the open edge at the growth front (see Figures S5 - S6). During the growth process, the catalyst melt drop slides along the already formed nanoribbon 'rail'. Increasing the GaSe supersaturation in the catalyst drop causes facile incorporation of dissolved GaSe into the open edge sites, thereby extending the nanoribbon length and pushing the drop forward. Increasing the ribbon thickness would require the nucleation of additional layers on the (001) plane, which appears to be kinetically suppressed. Hence, the GaSe nanoribbons are of constant thickness and atomically smooth over extended lengths, as shown by AFM analysis (Figures S7 - S8).

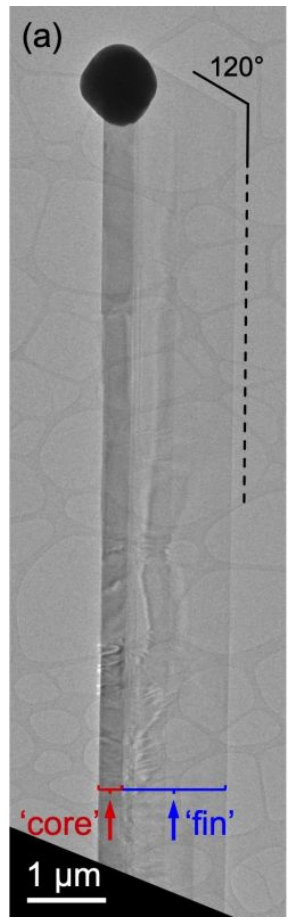

(b)

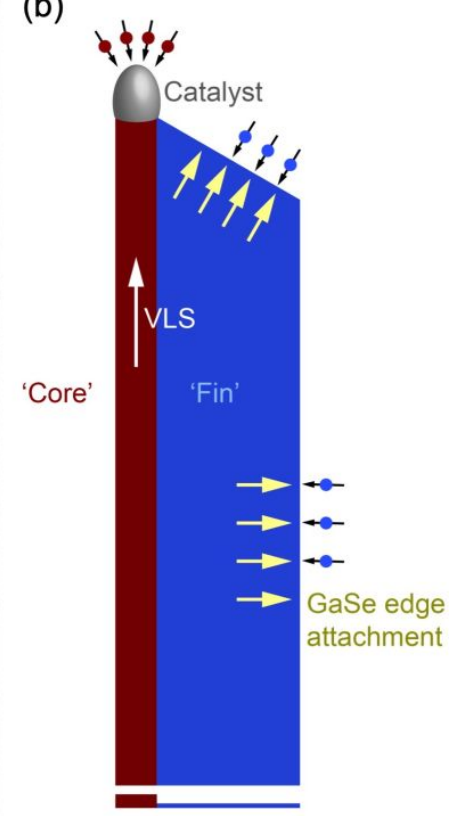

Figure S10. TEM analysis of a GaSe nanoribbon grown at $575^{\circ} \mathrm{C}$ over Ag catalyst. Note the contrast difference between a thick, VLS-grown GaSe nanoribbon 'core' matching the footprint of the VLS catalyst and a thinner lateral 'fin' that appears at higher growth temperatures due to nonspecific attachment of GaSe to one of the nanoribbon edges. The morphology shown here explains the distribution of photoluminescence intensity observed in Fig. 2 of the main text. 


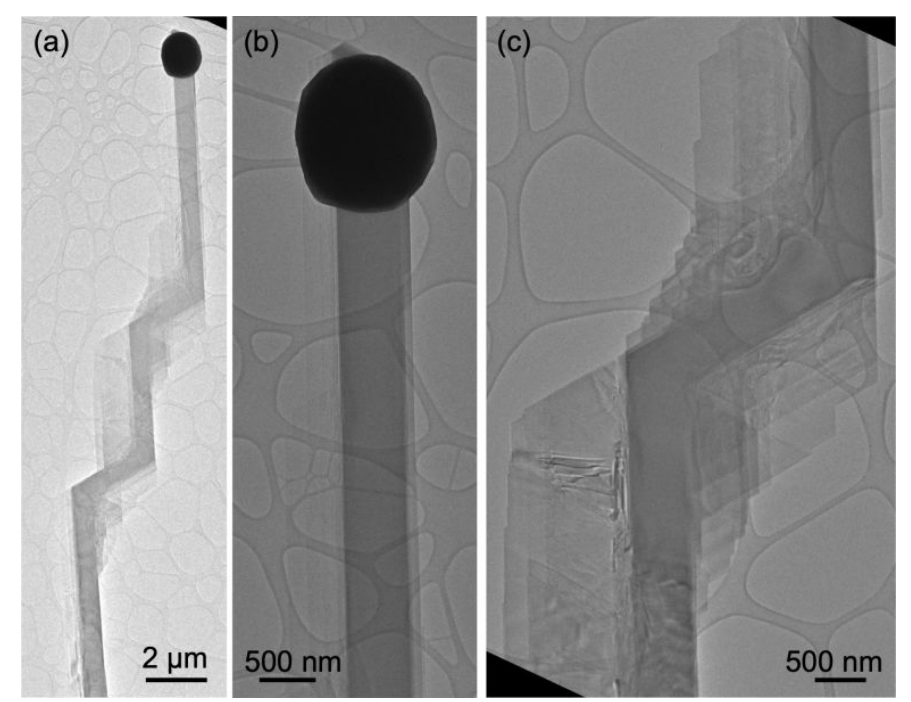

Figure S11. TEM of a multiply kinked GaSe nanoribbon grown at $575^{\circ} \mathrm{C}$ over $\mathrm{Ag}$ catalyst. Note the contrast difference between a thick, VLS-grown GaSe nanoribbon 'core' matching the footprint of the VLS catalyst and thinner lateral 'fins' that appear at higher growth temperatures due to non-specific attachment of GaSe to the nanoribbon edges.

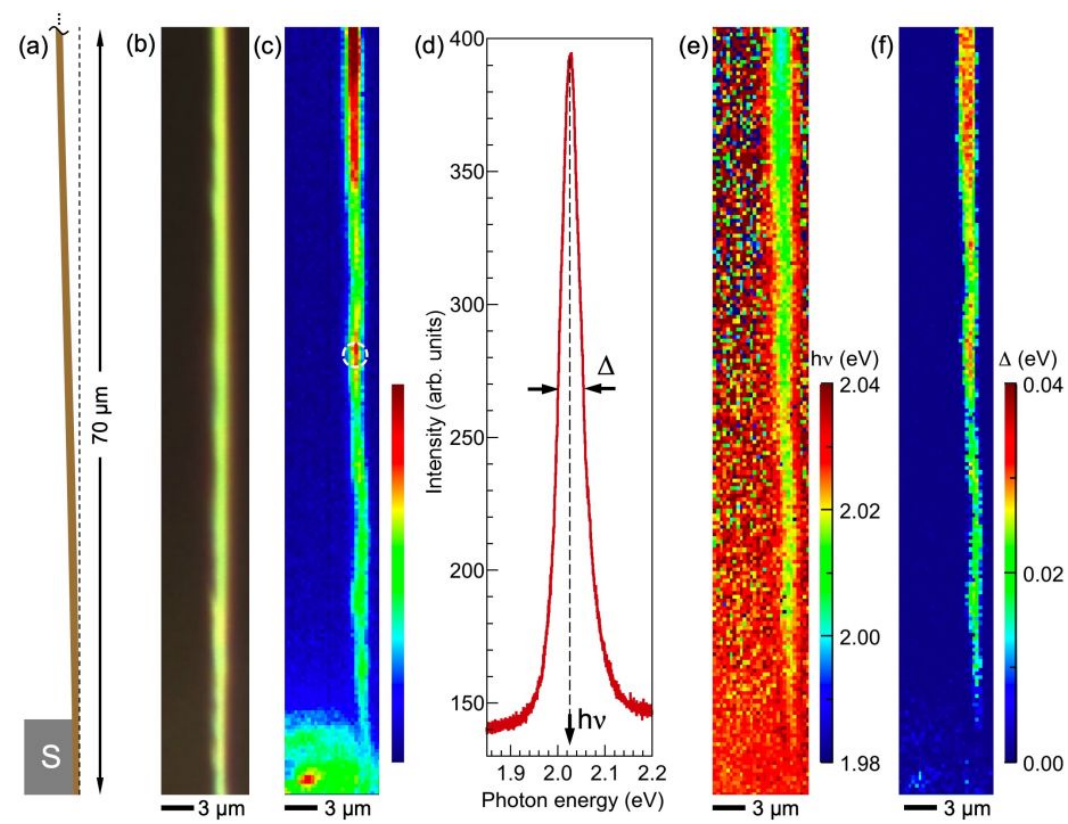

Figure S12. Photoluminescence spectroscopy and mapping of a long suspended GaSe nanoribbon. (a) Schematic of the geometry of the nanoribbon suspended off the edge of the growth substrate (S). (b) Optical microscopy of a $70 \mu \mathrm{m}$ long portion of the nanoribbon. (c) Photoluminescence intensity map of the nanoribbon segment shown in (b). (d) Photoluminescence spectrum obtained in the circled area in (c). (e) Map of the photoluminescence peak photon energy. (f) Map of the photoluminescence peak width. 


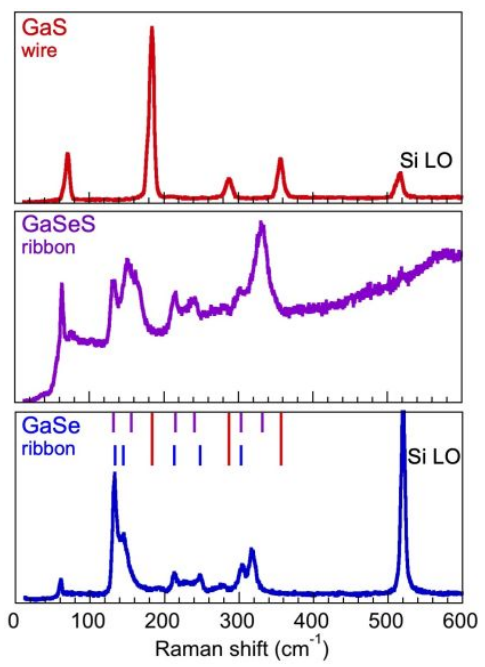

Figure S13. Comparison of Raman spectra of a GaSe nanoribbon (Fig. 2) with spectra of a GaSeS nanoribbon grown from GaSe and GaS precursors and a VLS GaS nanowire (Ref. 5). Note the systematic shifts to lower frequency of all phonon modes in GaSe compared to GaS. Smaller shifts of some of the modes are seen for the GaSeS nanoribbon, while other modes coincide with those in the GaSe ribbon. This suggests the coexistence of a GaSeS solid solution with pure GaSe. Finally, the doubling of some of the peaks in the nominally pure GaSe ribbon is consistent with the presence of a small amount of $S$ in a minority GaSeS solid solution phase coexisting with a majority GaSe phase. Colored vertical lines in the bottom panel mark the peak positions in the Raman spectra with the same colors (red: GaS; purple: GaSeS; blue: GaSe).
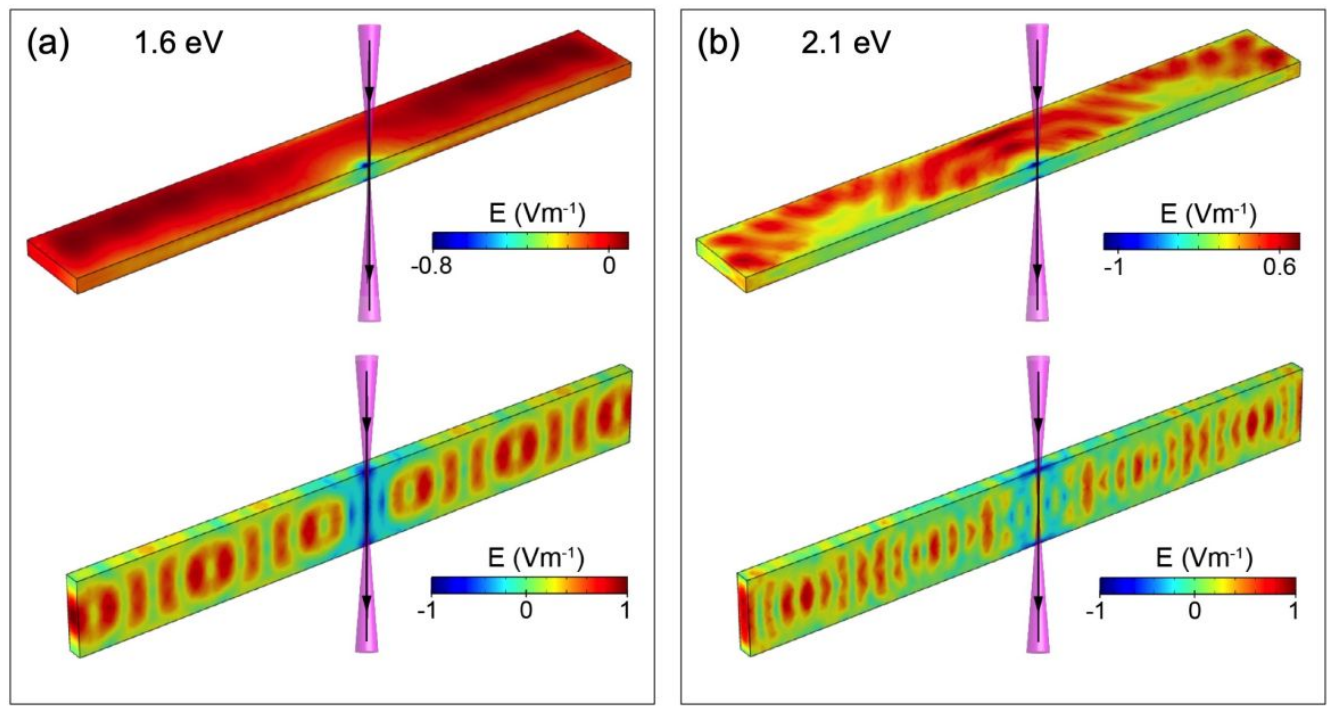

Figure S14. Simulation of propagating waveguide modes at two different energies in GaSe nanoribbons. (a) Numerical simulation of the excitation of a GaSe nanoribbon by the focused electron beam in STEM. Top: Electric field (energy: $1.6 \mathrm{eV}$ ) induced in a horizontal GaSe ribbon by the electron beam. Bottom: Induced electric field due to electron beam passage near a vertically standing GaSe nanoribbon. (b) Same as (a), but for $2.1 \mathrm{eV}$ energy. Geometry of the simulated GaSe nanoribbon: $4 \mu \mathrm{m}$ long, $500 \mathrm{~nm}$ wide, and $100 \mathrm{~nm}$ thick. In the simulations, the exciting electron beam is represented by a line current $10 \mathrm{~nm}$ outside the nanoribbon, as shown schematically in panels (a) and (b). 

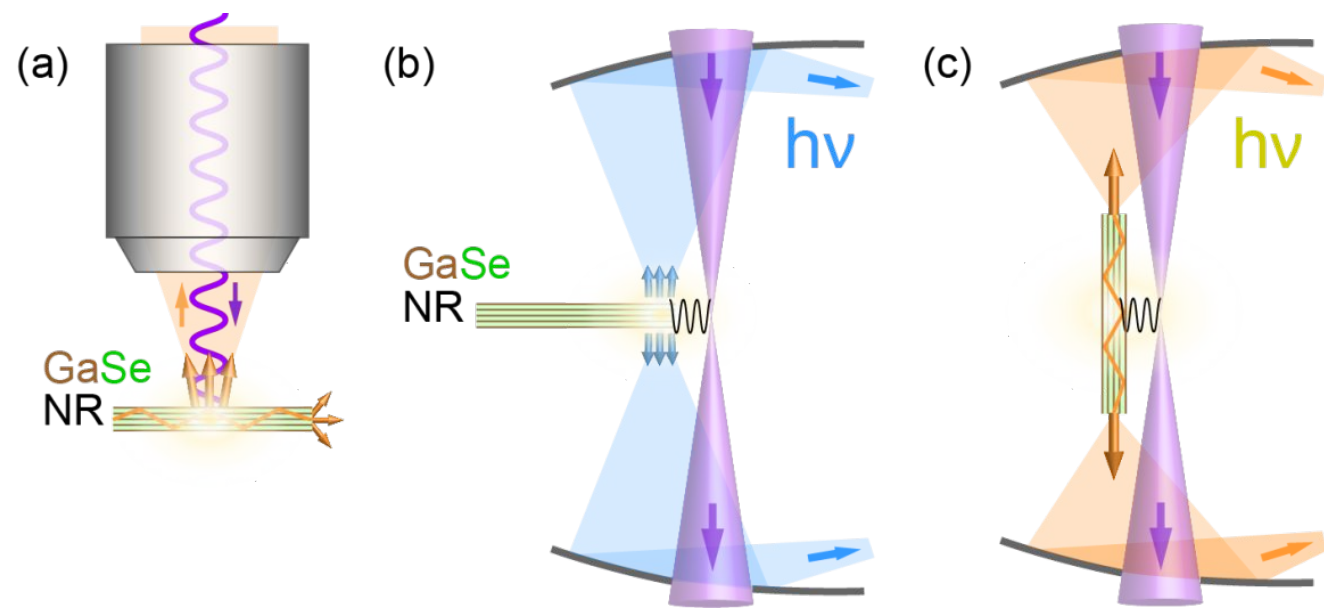

Figure S15. Comparison of luminescence excitation and detection in PL and STEM-CL. (a) Monochromatic laser excitation and confocal optical collection in micro-PL of a horizontal GaSe nanoribbon (NR). Note the launching of propagating modes in the ribbon by optical excitation, which leads to secondary emission at the nanoribbon side facets far from the focal spot (and hence not detected). ${ }^{6}$ (b) Excitation of a horizontal GaSe nanoribbon by the focused electron beam in STEM. Simulations (see Figure S14) show that significant electric fields are induced only close to the exciting electron beam, i.e., the collection optics sees only photons emitted from this local area (blue). (c) Excitation of a vertical GaSe nanoribbon by the focused electron beam. Simulations (see Figure S14) show the launching of propagating modes by the beam. This leads to light emission across the ribbon edge, which is detected by the CL collection optics (orange).
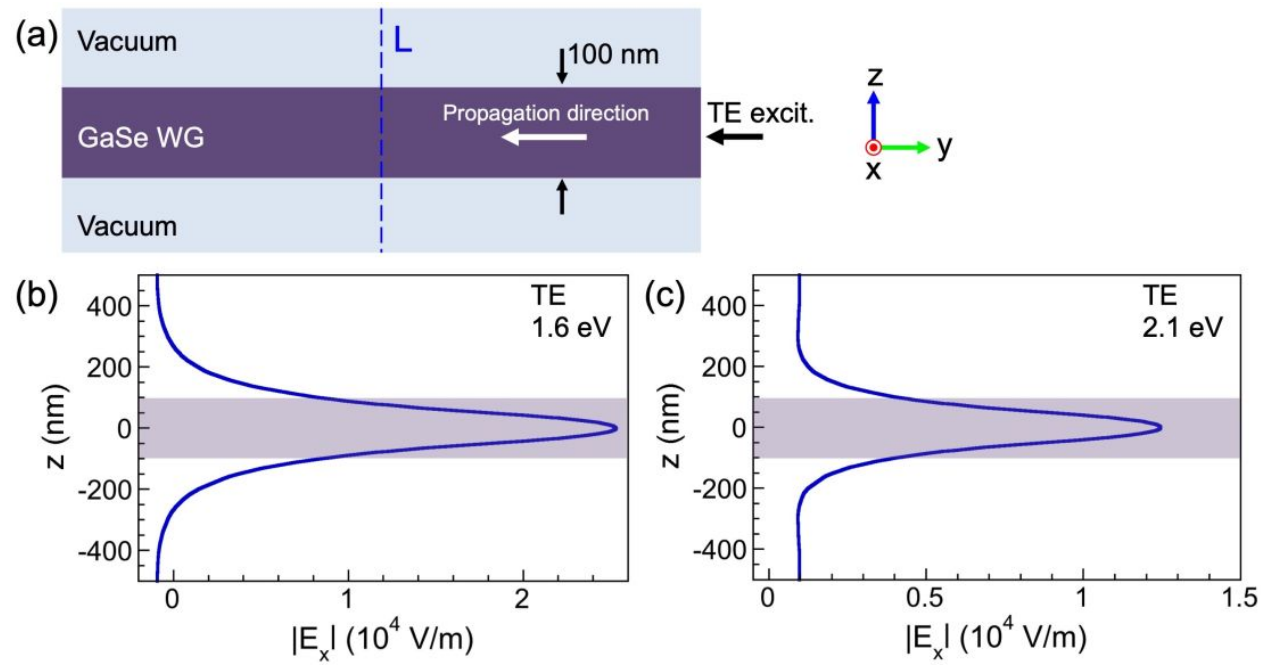

Figure S16. Propagating waveguide modes in GaSe nanoribbons. (a) Schematic of a symmetric waveguide consisting of a GaSe nanoribbon with vacuum cladding layers. Simulations were carried out for a $100 \mathrm{~nm}$ thick ribbon (matching the thickness observed in the experiments) by using a transverse electric (TE) mode incident from the left within the plane of the GaSe layers. (b), (c) Calculated electric field distribution for two different energies of the incident TE mode (1.6 $\mathrm{eV},(\mathrm{b}) ; 2.1 \mathrm{eV}(\mathrm{c})$ ) at position 'L' along the nanoribbon. Shaded areas indicate the thickness of the GaSe nanoribbon waveguide. The calculations clearly demonstrate the propagation of a TE mode along the GaSe ribbons. 
(a)

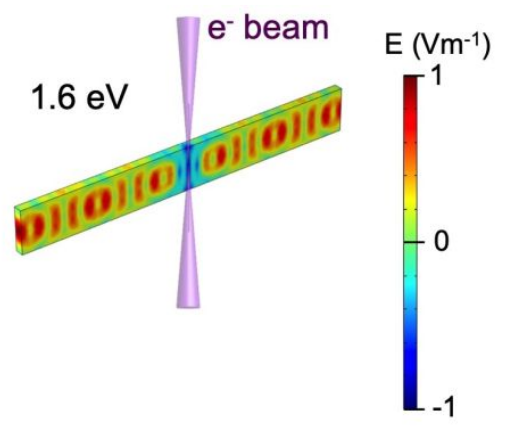

(b)

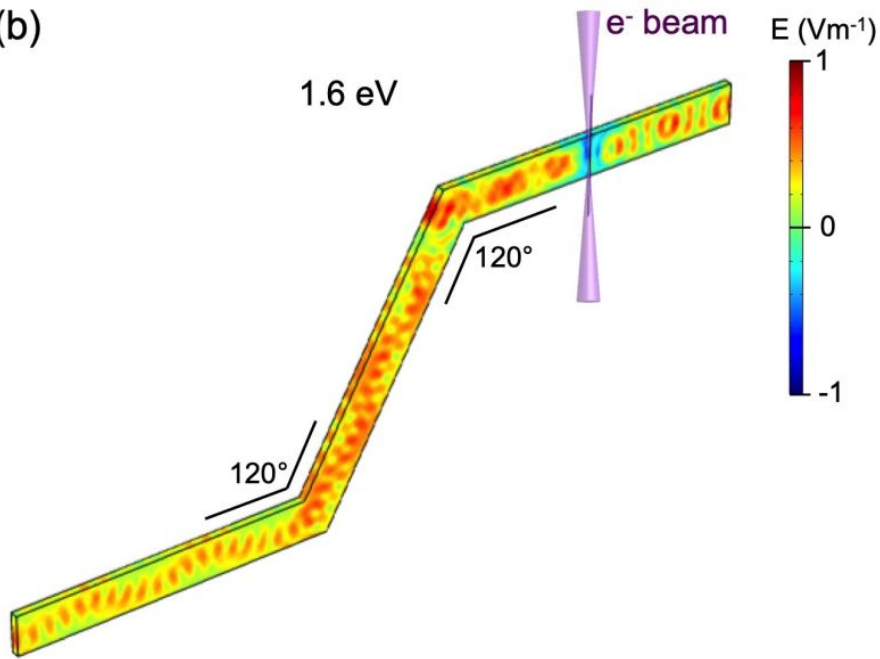

Figure S17. Comparison of simulations of propagating waveguide modes in straight and kinked GaSe nanoribbons. (a) Numerical simulation of the waveguide mode induced by the field of an aloof electron beam (energy: $1.6 \mathrm{eV}$ ). (b) Propagating waveguide mode induced in the same straight GaSe nanoribbon, which is now connected via two kinks to adjacent straight segments. The propagating mode, induced by an electron beam in the upper horizontal segment, propagates along the entire length of the kinked nanoribbon. Thickness of the simulated GaSe nanoribbon: $100 \mathrm{~nm}$. The ribbon in (a) and the upper horizontal section in (b) are $4 \mu \mathrm{m}$ long.

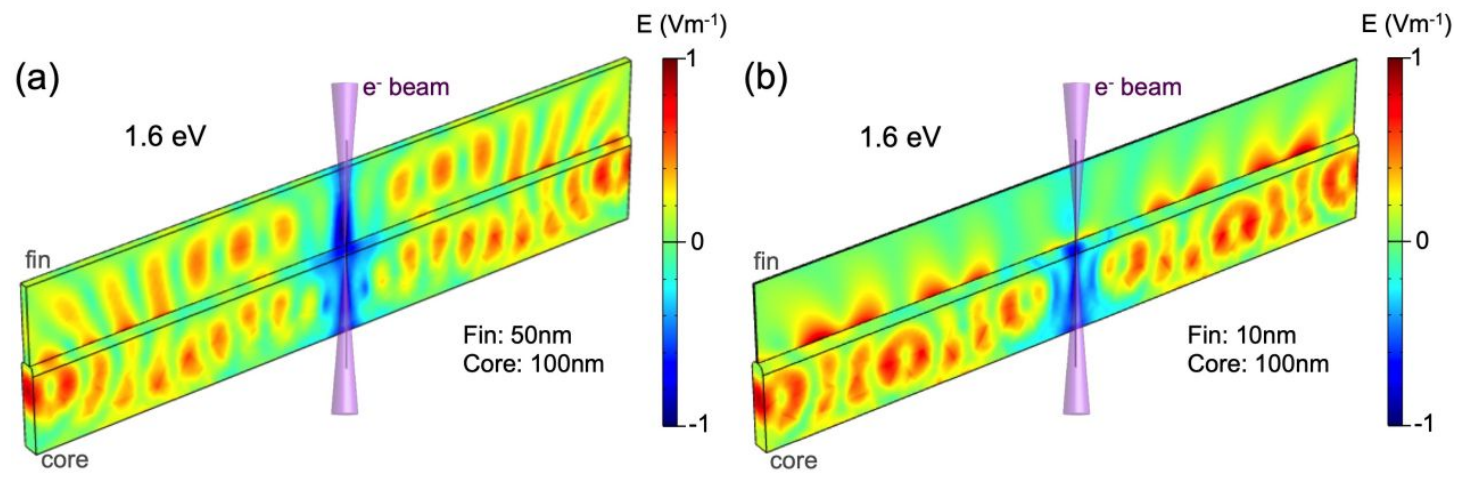

Figure S18. Comparison of simulations of propagating waveguide modes in "core-fin" nanoribbons with different thickness of the attached fin. (a) Numerical simulation of the waveguide mode induced by the field of an aloof electron beam (energy: $1.6 \mathrm{eV}$ ), for a core-fin nanoribbon with $100 \mathrm{~nm}$ core thickness and $50 \mathrm{~nm}$ fin thickness. Similar results are found for fins with thickness $>30 \mathrm{~nm}$. (b) Numerical simulation of the waveguide mode induced by the field of an aloof electron beam (energy: $1.6 \mathrm{eV}$ ), for a core-fin nanoribbon with $100 \mathrm{~nm}$ core thickness and $10 \mathrm{~nm}$ fin thickness. While the electron beam induces a propagating mode in both the core and fin at $50 \mathrm{~nm}$ fin thickness, the $10 \mathrm{~nm}$ fin does not support a long-range propagating mode so that it only experiences evanescent fields that decay from the core-fin junction into the ultrathin fin. Length of the simulated ribbons: $4 \mu \mathrm{m}$. 

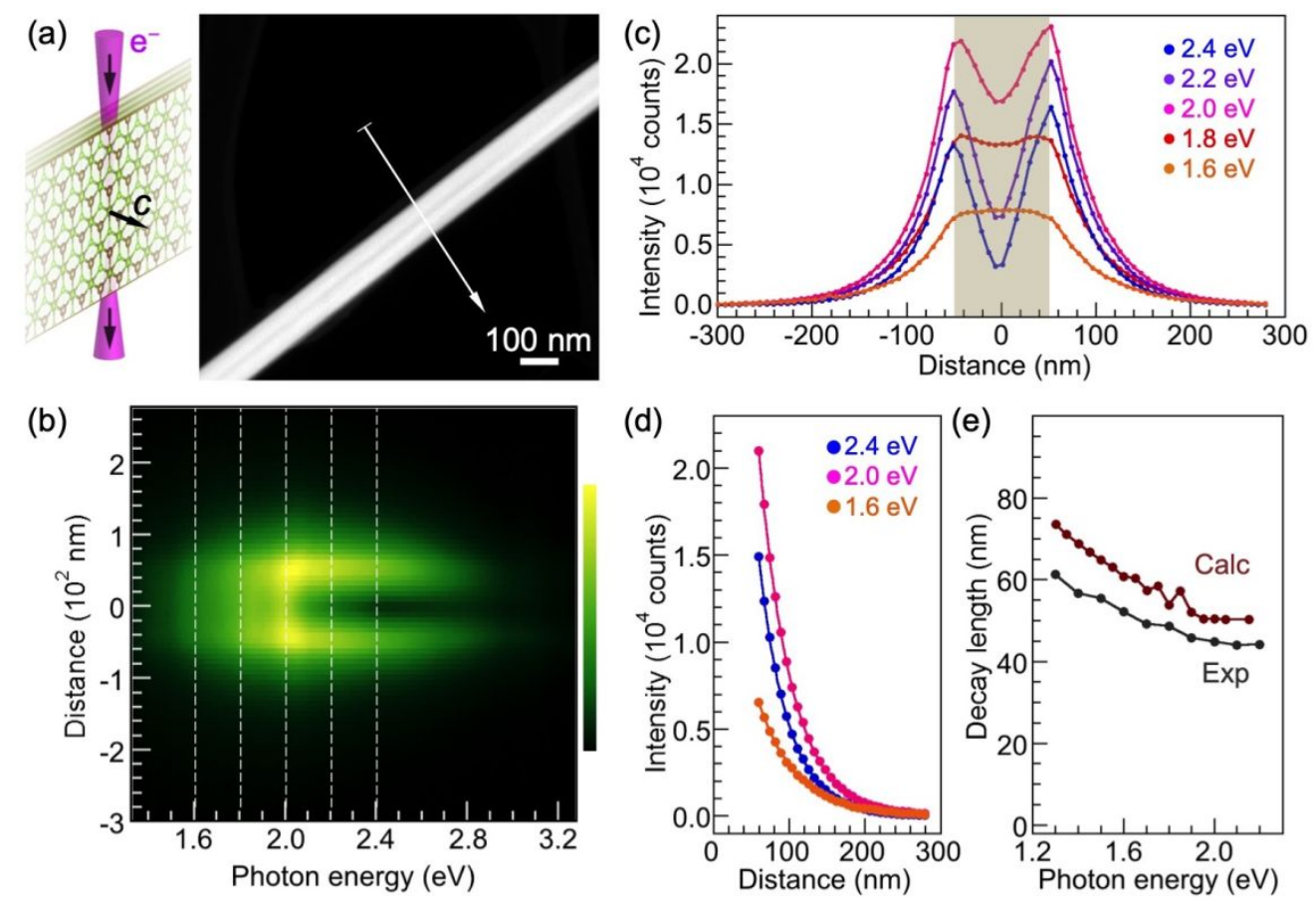

Figure S19. Probing evanescent fields by STEM-CL and simulations. (a) Schematic and HAADF-STEM image of a vertical GaSe nanoribbon (see also Figure 5 of the main text). (b) Hyperspectral STEM-CL linescan along the arrow shown in (a). (c) STEM-CL intensity profiles along the dashed lines in (b), i.e., for free-space photon energies of $1.6 \mathrm{eV}, 1.8 \mathrm{eV}, 2.0 \mathrm{eV}, 2.2$ $\mathrm{eV}$, and $2.4 \mathrm{eV}$. The shaded area around the origin represents the physical thickness $(\sim 100 \mathrm{~nm})$ of the vertical ribbon. (d) Examples of the $C L$ intensity decay into the vacuum adjacent to the GaSe nanoribbon. Symbols: Measured data; lines: Fitted exponential decay profiles. (e) Plot of the 1/e decay length as a function of free-space photon energy, compared with decay lengths obtained in numerical simulations. The experimental values ('Exp') show the same increase in the decay length with decreasing energy but are generally $\sim 15 \%$ smaller than the calculated ones ('Calc').

\section{Supplementary References}

1. Le Toullec, R.; Piccioli, N.; Mejatty, M.; Balkanski, M. Optical constants ofe-GaSe. Il Nuovo Cimento B (1971-1996) 1977, 38, 159-167.

2. Adachi, S.; Shindo, Y. Optical constants of $\varepsilon$-GaSe. Journal of Applied Physics 1992, 71, 428431.

3. Choi, S. G.; Levi, D. H.; Martinez-Tomas, C.; Sanjosé, V. M. Above-bandgap ordinary optical properties of GaSe single crystal. Journal of Applied Physics 2009, 106, 053517.

4. Sutter, P.; Khorashad, L. K.; Argyropoulos, C.; Sutter, E. Cathodoluminescence of Ultrathin Twisted $\mathrm{Ge}_{1-\mathrm{x}} \mathrm{Sn}_{\mathrm{x}} \mathrm{S}$ van der Waals Nanoribbon Waveguides. Advanced Materials 2021, 33, 2006649.

5. Sutter, E.; French, J. S.; Sutter, S.; Idrobo, J. C.; Sutter, P. Vapor-Liquid-Solid Growth and Optoelectronics of Gallium Sulfide van der Waals Nanowires. ACS Nano 2020, 14, 6117-6126. 
6. Suzuki, M.; Kohda, M.; Takasuna, S.; Matsuzaka, S.; Sato, Y.; Tanabe, T.; Oyama, Y.; Nitta, J. Effect of optical waveguide on photoluminescence polarization in layered material GaSe with millimeter scale. Japanese Journal of Applied Physics 2018, 57, 020308. 